Article

\title{
Doping Liquid Crystal Cells with Photocurable Monomer via Holographic Exposure to Realize Optical-Scattering-Free Infrared Phase Modulators with Fast Response Time
}

\author{
Chun-Yu Chien and Chia-Rong Sheu * (D) \\ Department of Photonics, National Cheng Kung University, Tainan 701, Taiwan; jun026103@hotmail.com \\ * Correspondence: pizisheu@mail.ncku.edu.tw; Tel.: +886-6-275-7575 (ext. 63929)
}

Academic Editor: Wei Lee

Received: 30 May 2017; Accepted: 4 July 2017; Published: 7 July 2017

\begin{abstract}
Photocurable monomer-doped liquid crystal (LC) cells were processed via holographic exposure using a low-power He-Ne laser to generate holographic polymer networks. The polymer network LC (PNLC) cells are used to fabricate infrared phase modulators at $1550 \mathrm{~nm}$ wavelength possessing favorable electro-optical performance. Compared with our previous work, the percentages of ingredients in the LC mixture filled in PNLC cells underwent a slight change. The $2 \mathrm{wt} \%$ concentration of anisotropic monomer RM257 were in place of isotropic monomer $\mathrm{N}$-vinyl-2-pyrrolidinone (NVP). As a result, the fabricated phase modulators also maintained well homogeneous LC alignments and optical-scattering-free characteristics. Furthermore, NVP dopant successfully reduced the operating voltages from $95 V_{\text {rms }}$ to $79 V_{\text {rms }}$ to prevent polymer network deformation when electrically operating with higher voltages. The fabricated infrared phase modulators had a good average response time (i.e., rising time of $0.88 \mathrm{~ms}$ and falling time of $0.40 \mathrm{~ms}$ ).
\end{abstract}

Keywords: holographic exposure; liquid crystal; infrared phase modulator

\section{Introduction}

To date, nematic liquid crystals (NLCs) are very popular and useful electro-optical materials due to their dielectric anisotropy and optical birefringence, which are sensitive to LC alignments, environmental temperature, and driving methods. They are widely applied in various electro-optical devices including spatial light modulators, LC lenses, and displays [1-3]. For typical optical phase modulation, a homogeneous LC cell is usually adopted to provide maximum phase retardation $(\delta)$ using the equation $\delta=2 \pi \Delta n d / \lambda$, where $\Delta n$ is LC birefringence, $d$ is cell gap, and $\lambda$ is incident wavelength [4]. Considering that NLCs cells are used as infrared phase modulators, the cell gap usually has a value larger than $10 \mu \mathrm{m}$ to achieve a completed $2 \pi$ phase modulation. However, the large thickness of the LC gap is unfavorable for optical response time in LC electro-optical devices except for several other reasons related to LC elastic constants, LC rotational viscosity [5,6], and electrically driving methods. Usually, the rising time can be sped up with higher operating voltages. However, the falling time is directly related to the relaxation of LC reorientations and is only controlled by restoring elastic torque. Therefore, the thicker cell gaps have a slow falling time. Recently, polymer network LC (PNLC) cells have been widely studied and demonstrated a fast response time [7-11]. In this method, the molecules of an anisotropic monomer like RM257 possessing an LC building block structure are usually doped with LCs and processed with UV exposure, so that the generated polymer networks provide constraints to LCs to effectively speed up the falling time during the relaxation of LC molecular reorientations. However, light scattering occurs due to the refractive index mismatch of 
polymer networks and LC microdomains [1]. The degree of light scattering can be reduced by using much smaller LC microdomains via optimal processing conditions such as monomer concentrations and photopolymerization conditions [9-13]. Several studies have demonstrated scattering-free PNLC phase modulators at near infrared wavelength [14-17].

Holographic exposure based on optical interference spatially generates very fine and directionally periodic interference patterns with a pitch of a few hundred nanometers. Many studies used this process to fabricate optical-scattering-free electro-optical LC devices such as holographic polymer-dispersed LCs [18] and polymer LC polymer slices [19]. In our previous work, we adopted the holographic exposure via a low-power He-Ne laser to expose the anisotropic monomer RM257-doped LC cells to generate holographic polymer networks and to realize a LC phase modulator with low light scattering and ultra-fast response time at visible wavelengths [13]. However, the operating voltage $\left(185 \mathrm{~V}_{\mathrm{rms}}\right)$ was not suitable for conventional operations. In addition, high operating voltages usually induce an electrostriction effect, that is, electrical deformations of anisotropic polymer networks [20,21], which degrades device performance, such as unstable phase modulations with respect to electrical operations. In the present study, PNLC cells processed via holographic exposure were also used to fabricate optical-scattering-free infrared phase modulators with a fast response time. Furthermore, we report a method to reduce operating voltages of infrared phase modulators by varying the ratio of RM257 and N-vinyl-2-pyrrolidinone (NVP). We found that increasing the percentage of NVP dopants successfully reduces operating voltages and the electrostriction effect.

\section{Experimental}

The LC mixture filled in empty cells ( $15 \mu \mathrm{m}$ gap thickness, purchased from Chipset Technology, Miaoli, Taiwan) for homogeneous LC cells contained the following: NLCs (E7, Daily Polymer, Kaohsiung, Taiwan), anisotropic monomer (RM257, HCCH, Yangzhong, China), photo-initiator (H-Nu-Blue-640, Spectra Group Limited, Millbury, USA), co-initiator (Borate-V, Spectra Group Limited, Millbury, USA), and mono-acrylate monomer (NVP, Sigma-Aldrich, St. Louis, USA). Table 1 shows ingredient ratios of LC mixture filled in experimental PNLC cells for processing holographic exposure. The ingredients of $\mathrm{H}-\mathrm{Nu}-\mathrm{Blue}-640$ and Borate- $\mathrm{V}$ were used to initiate photopolymerization of LC cells when exposed with a He-Ne laser. The anisotropic monomer of RM257 with a rod-like molecular unit possesses behaviors of an LC phase at a temperature range from $70{ }^{\circ} \mathrm{C}$ to $126{ }^{\circ} \mathrm{C}$, which is usually photopolymerized with a growth direction of polymer networks along with the direction of LC alignments. NVP was used as a homogenizer to mix H-Nu-Blue-640 and Borate-V to yield a uniform precursor. Thereafter, E7 and RM257 are mixed with the precursor to complete the mixing process of the LC mixture. Furthermore, NVP was also considered as the photocurable monomer and varied percentages with respect to RM257 to improve electro-optical performance of PNLC cells. Except E7, the chemical structures of all other ingredients are shown in [22]. Figure 1a illustrates the setup for processing holographic exposure, where a red He-Ne laser beam $(\lambda=632.8 \mathrm{~nm}$, LASOS, Jena, Germany) serially passed through the attenuator, polarizer, and beam expander to achieve an s-polarization light beam with controllable intensity and optical quality. Holographic exposure processes were executed by means of two coherent beams from the previous s-polarization beam passing through a beam splitter with equal power intensity $\left(0.1 \mathrm{~mW} / \mathrm{cm}^{2}\right)$ to expose the LC cell. Both interference beams were incident to the LC cell with an angle of $160^{\circ}$ between their wave vectors to generate the interference patterns with pitch of $209 \mathrm{~nm}$ as shown in Figure 1b. Due to specific molecular characteristics, the RM257 and NVP monomers were forced to move toward the regions with higher light intensity during holographic exposure processes. Therefore, the interference patterns generated by holographic exposure will possibly lead to the formed morphology of holographic polymer networks in PLNC cells as schematically shown in Figure 1c. Finally, the smaller LC domains are embedded in polymer networks to achieve light scattering reduction [13]. 
Table 1. Ingredient percentages of the LC mixture filled in experimental PNLC cells for processing holographic exposure.

\begin{tabular}{cccccc}
\hline Label of PNLC Cells & $\begin{array}{c}\text { RM257 } \\
\mathbf{( w t / \% )}\end{array}$ & $\begin{array}{c}\text { NVP } \\
\mathbf{( w t} \%)\end{array}$ & $\begin{array}{c}\text { E7 } \\
\mathbf{( w t \% )}\end{array}$ & $\begin{array}{c}\text { H-Nu-Blue-640 } \\
\mathbf{( w t \% )}\end{array}$ & $\begin{array}{c}\text { Borate-V } \\
\text { (wt\%) }\end{array}$ \\
\hline RM8 & 8 & 2 & 89.5 & 0.25 & 0.25 \\
RM7 & 7 & 2 & 90.5 & 0.25 & 0.25 \\
RM6 & 6 & 2 & 91.5 & 0.25 & 0.25 \\
RM5 & 5 & 2 & 92.5 & 0.25 & 0.25 \\
RM5N2 & 5 & 4 & 90.5 & 0.25 & 0.25 \\
\hline
\end{tabular}

(a)

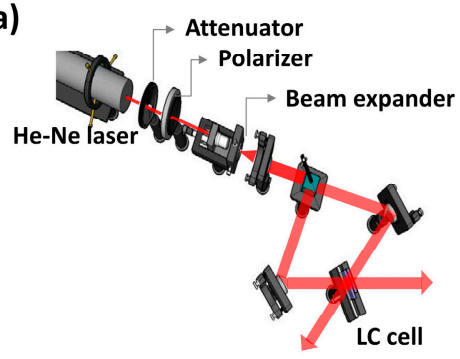

(b)

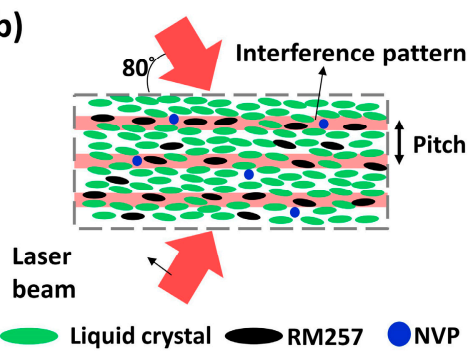

(c)

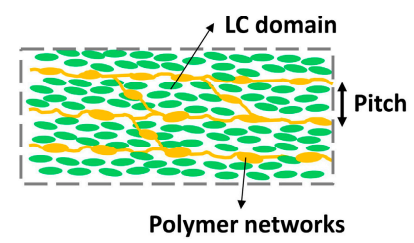

Figure 1. (a) Experimental setup for processing holographic exposure; (b) Schematic illustration of holographic interference patterns generated in cell to expose the materials of RM257 and NVP to be photopolymerized; (c) Schematic illustration of holographic polymer networks formed in the cell after holographic exposure processes.

Figure 2 shows the setup used to measure the relative curves of voltage versus optical reflectance (V-R) of completely exposed PNLC cells. In the experiment, we utilized a mirror reflector to reflect the normally incident laser beam ( $\lambda=1550 \mathrm{~nm}$, THORLABS, Newton, USA) passing through the PNLC cell once. A detector was used to record the final light intensity after passing the PNLC cell twice with respect to applying various $\mathrm{AC}$ voltages $(10 \mathrm{kHz}$ square waveform) in PNLCs.

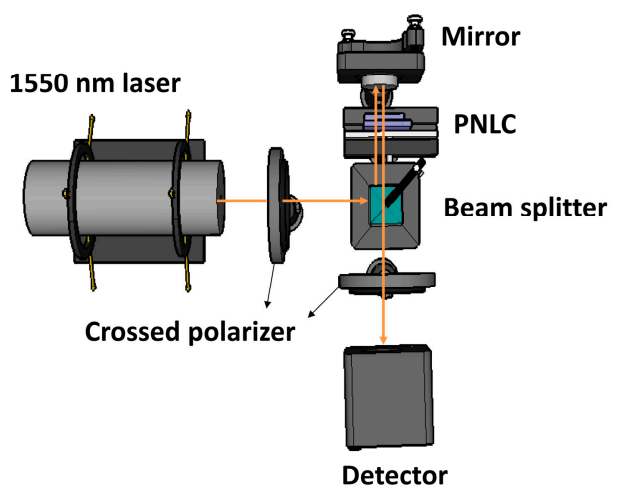

Figure 2. Experimental setup for measuring the V-R curves of completed PNLC cells. The rubbing direction of the cell is $45^{\circ}$ with respect to the polarization direction of the crossed polarizers. 


\section{Results and Discussion}

Firstly, intensity loss due to light scattering in the PNLC cells was measured. Without a pair of crossed polarizers, a laser beam $(\lambda=1550 \mathrm{~nm})$ was normally incident to the completed PNLC cell located $20 \mathrm{~cm}$ away from the laser position. An iris was used to prevent scattering light from entering the detector and permit only light intensity along the initial incident direction. The measured transmittance in different PNLC cells with respect to various applied voltages is shown in Figure 3a, where transmittance in percentage was the transmittance ratios of cells versus the reference LC cell filled with only E7 LCs. The completed PNLC cells with various concentrations of RM257 dopants also possessed well and uniform homogeneous LC alignments as shown in the inserts of Figure 3a, which are very different from the polymer-dispersed LC cells [23]. Therefore, all four PNLC cells show good transmittance as high as $97 \%$ without applied voltage. A slight decrease of transmittance compared with the cells filled with E7 LCs is from the mismatch of refractive indices, because the ratios of refractive indices between E7 LCs and RM257 are $n_{\mathrm{o}, \mathrm{E} 7}=1.521 / n_{\mathrm{e}, \mathrm{E} 7}=1.747$ and $n_{\mathrm{o}, \mathrm{RM} 257}$ $=1.508 / n_{\mathrm{e}, \mathrm{RM} 257}=1.687$ at $\lambda=589 \mathrm{~nm}$, respectively. When electrically operating completed PNLC cells, the micro size LC domains appeared that induced the light scattering in the LC cell, but the samples RM7 and RM8 maintained the high transmittance even at a voltage of $140 \mathrm{~V}_{\mathrm{rms}}$. Although, using holographic exposure to process LC cells usually improves light scattering, positive results are also related to the concentrations of the RM257 dopant. If RM257 is used with too low a concentration, it will not decrease light scattering as shown by the experimental results of the RM5 and RM6 cells. Figure $3 \mathrm{~b}$ shows the measured V-R curves for four experimental PNLC cells, where optical reflectance was normalized to the maximum reflectance in a reference cell filled with only E7 LCs. Generally, the generated polymer networks after holographic exposure in the PNLC cells provide more constrains to LC molecules to increase operating voltages $V_{2 \pi}$ for $2 \pi$ phase modulation and $V_{\text {th }}$ for threshold voltages. Furthermore, the voltages of $V_{2 \pi}$ and $V_{t h}$ are mainly related to the morphology of the polymer networks and the size of LC microdomains [24,25]. Especially, the value of $V_{\text {th }}$ approximately satisfied

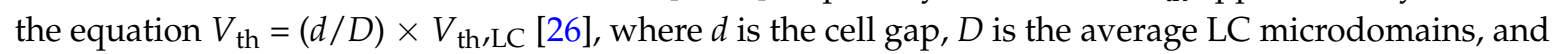
$V_{\text {th,LC }}$ is the threshold voltages of cells filled with LCs. By referred to [24,25], the higher concentrations of monomer dopant in cells usually generate smaller LC microdomain sizes. Therefore, the values of $V_{2 \pi}$ and $V_{\text {th }}$ are increased in the PNLC cells with higher concentrations. Although the RM7 and RM8 PNLC cells show good performance of V-R curves, the voltages of phase modulation $V_{2 \pi}$ are also too high for ideal operations, which are $98 \mathrm{~V}_{\mathrm{rms}}$ and $123 \mathrm{~V}_{\mathrm{rms}}$, respectively.

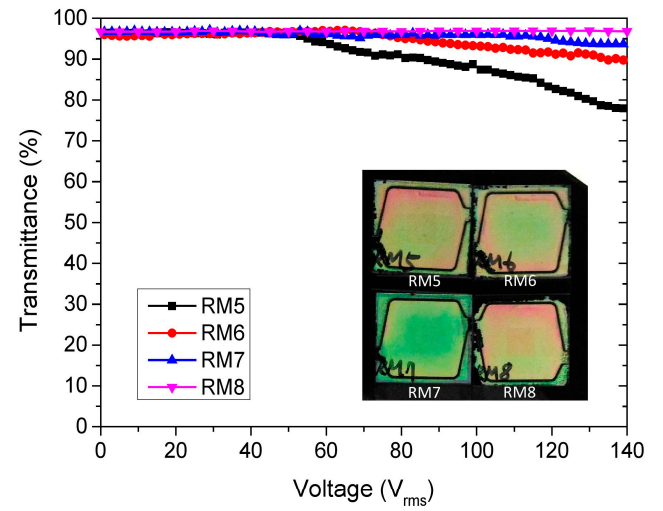

(a)

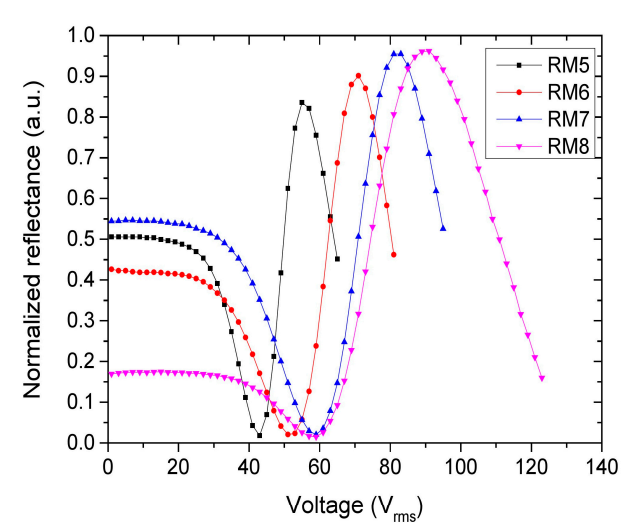

(b)

Figure 3. Measurements of transmittance and reflectance with respect to applied voltages in completed PNLC cells with various RM257 concentrations: (a) the measurements of transmittance versus applied voltages. The inserts show optical observations of four completed PNLC cells via the light box with a pair of crossed polarizers; (b) the measurements of normalized reflectance curves versus applied voltages in the same cells via the setup shown in Figure 2. 
The PNLC cell labeled RM5 possessed a low phase modulation voltage of $65 \mathrm{~V}_{\text {rms }}$ but suffered more serious light scattering and inaccurate phase modulation control. Therefore, further investigations were based on the conditions of the RM5 cell to improve optical performance. Due to considerations of NVP as the photocurable monomer with various percentages instead of RM257 to improve performance of PNLC cells, we doped more than $2 \mathrm{wt} \%$ NVP instead of RM257 in the previous RM5 cell and labeled it RM5N2 to possibly induce a different polymer network morphology. Figure 4a shows the comparisons of transmittances in RM7, RM5, and RM5N2 PNLC cells. Obviously, the RM5N2 cell showed very similar results to the RM7 cell, the more than $2 \mathrm{wt} \%$ NVP doped in the RM5N2 cell possibly achieved much smaller LC microdomains than the RM5 cell but close to the microdomain sizes in the RM7 cell. Compared with the RM257 dopant with LC building block structure, the NVP dopant has no such structure to provide homogeneous LC alignments. However, doping the RM5N2 cell with more than $2 \mathrm{wt} \%$ NVP still assisted and maintained a uniform LC alignment as shown in the insert of Figure 4a. Figure $4 \mathrm{~b}$ shows the comparisons of measured V-R curves in RM5 and RM5N2 cells, where the phase modulation voltage was $79 \mathrm{~V}_{\text {rms }}$ in the RM5N2 cell, which was higher than the RM5 cell. A high phase modulation voltage was needed due to the much smaller LC microdomains in the RM5N2 cell. Figure 5 shows the profiles of phase modulation versus applied voltages in RM8, RM7, and RM5N2 PNLC cells as infrared phase modulators. The major phase modulation voltages are listed and summarized in Table 2. As a result, the RM5N2 cell possessed a low phase modulation voltage due to the more than $2 \mathrm{wt} \% \mathrm{NVP}$ dopant, which reduced constrains between LC molecules and generated polymer networks.

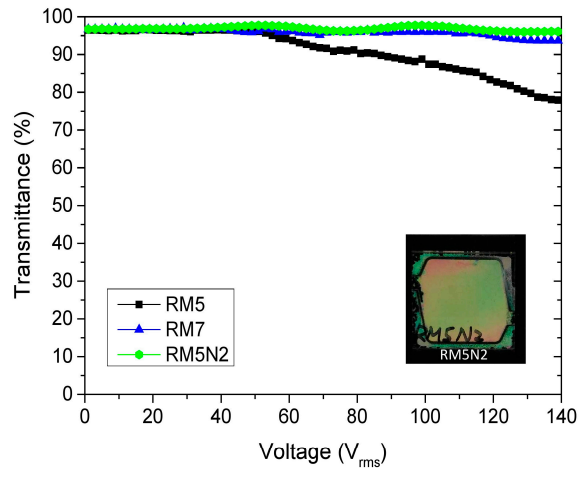

(a)

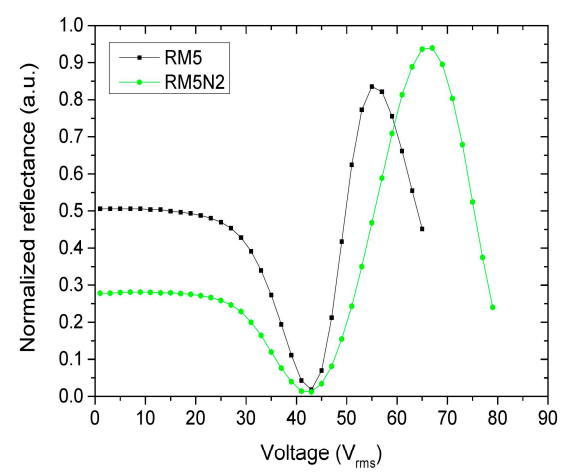

(b)

Figure 4. Measurements of transmittance and reflectance with respect to applied voltages in RM5, RM7, and RM5N2 PNLC cells: (a) Comparisons of transmittance and optical observations in the RM5N2 cell via the light box with a pair of crossed polarizers; (b) Comparisons of normalized reflectance versus applied voltages in RM5 and RM5N2 cells.

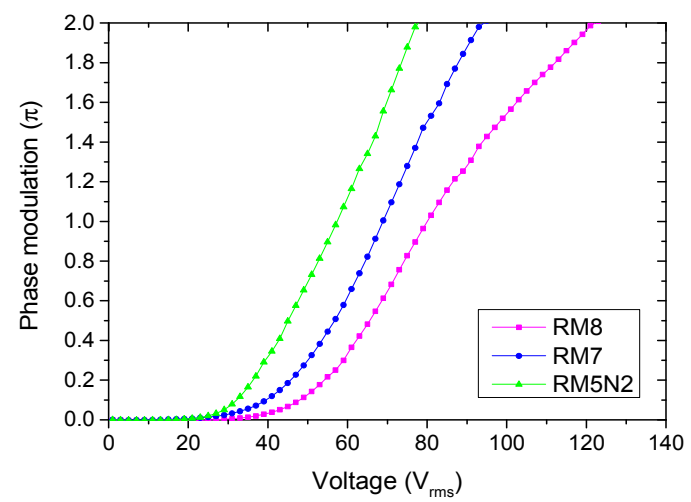

Figure 5. Comparisons of $2 \pi$ phase modulation in RM7, RM8, and RM5N2 cells as infrared phase modulators for the light beam wavelength of $\lambda=1550 \mathrm{~nm}$. 
Table 2. Typical voltages of phase modulations in the three fabricated phase modulators.

\begin{tabular}{ccccc}
\hline Label of PNLC Cells & $V_{\mathbf{0 . 5} \boldsymbol{\pi}}\left(\mathrm{V}_{\text {rms }}\right)$ & $V_{\mathbf{1 . 0} \boldsymbol{\pi}}\left(\mathrm{V}_{\mathbf{r m s}}\right)$ & $V_{\mathbf{1 . 5 \pi}}\left(\mathrm{V}_{\mathbf{r m s}}\right)$ & $V_{\mathbf{2 . 0} \boldsymbol{\pi}}\left(\mathrm{V}_{\mathbf{r m s}}\right)$ \\
\hline RM8 & 66 & 80 & 98 & 120 \\
RM7 & 57 & 69 & 80 & 95 \\
RM5N2 & 45 & 59 & 68 & 79 \\
\hline
\end{tabular}

Except for the previous statements to describe characteristics of the fabricated infrared phase modulators, their optical response time was further measured and described in the following. According to experimental results in Figure 5 and Table 2, the completed phase modulators (i.e., RM7, RM8, and RM5N2 cells) were electrically switched between on and off states with respect to various applied voltages corresponding to individual phase modulations (i.e., $0.5 \pi, 1.0 \pi, 1.5 \pi$, and $2.0 \pi)$ in the $4 \mathrm{~ms}$ duration. Figure 6a shows the response time of phase modulations in the RM8 cell with respect to four applied voltages. Some ideal values of modulated phase were not achieved if phase modulation voltages were applied according to the data in Figure 5, including the switching voltages of $V_{1.0 \pi}, V_{1.5 \pi}$, and $V_{2.0 \pi}$. This issue was mainly attributed to the electrostriction effect of polymer networks. We obtained the phase modulation voltage $V_{2 \pi}$ in PNLC cells via measurements of V-R curves and $1 \mathrm{~s}$ waiting time for each data point. During measurements of V-R curves, the anisotropic polymer networks generated by the RM257 dopant were continuously deformed due to higher electrical voltages, which also provided the phase variations in PNLC cells. Due to short operation time in real phase modulations, the polymer networks were not seriously deformed to induce unstable phase modulation control as previously described. The ideal and correct phase modulations can be achieved with higher applied voltages [13] or a longer waiting time (about a few seconds) [20]. In this study, we applied voltages of 84,110 , and $135 \mathrm{~V}_{\text {rms }}$ to ideally achieve the $1.0 \pi, 1.5 \pi$, and $2.0 \pi$ phase modulations as shown in Figure 6b. Thereafter, measurements of response time, including rising and falling time, were based on applied voltages corresponding to $0.5 \pi, 1.0 \pi, 1.5 \pi$, and $2.0 \pi$ phase modulations. The rising time $\left(\tau_{r}\right)$ is defined as the time spent for $0 \%$ to $90 \%$ phase modulation, and falling time $\left(\tau_{\mathrm{f}}\right)$ is defined as the time spent for $100 \%$ to $10 \%$ phase modulation. Response time, phase deviations, and required boost voltages are summarized in Table 3.

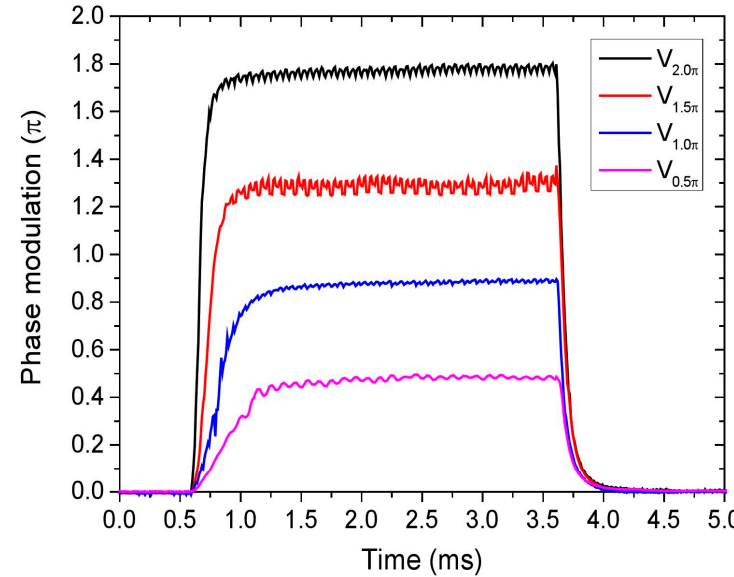

(a)

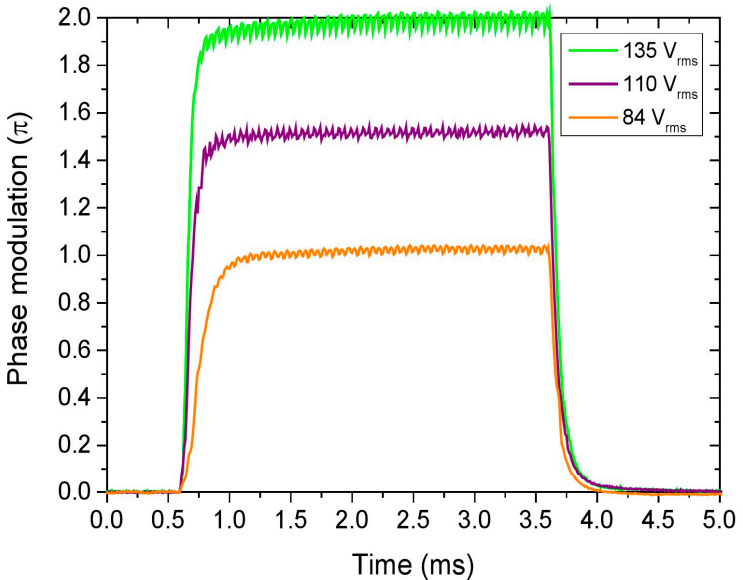

(b)

Figure 6. Measurements of response time in the RM8 cell for operating phase modulations: (a) Phase modulation voltages according to data in Table 2; (b) High phase modulation voltages corresponding to correct phase modulations of $1.0 \pi, 1.5 \pi$, and $2.0 \pi$. 
Table 3. Summary of phase deviations, response time, and the required boost voltages in three phase modulators with correct phase control.

\begin{tabular}{|c|c|c|c|c|c|c|c|c|c|c|c|c|}
\hline & \multicolumn{4}{|c|}{ RM8 } & \multicolumn{4}{|c|}{ RM7 } & \multicolumn{4}{|c|}{ RM5N2 } \\
\hline & $V_{0.5 \pi}$ & $V_{1.0 \pi}$ & $V_{1.5 \pi}$ & $V_{2.0 \pi}$ & $V_{0.5 \pi}$ & $V_{1.0 \pi}$ & $V_{1.5 \pi}$ & $V_{2.0 \pi}$ & $V_{0.5 \pi}$ & $V_{1.0 \pi}$ & $V_{1.5 \pi}$ & $V_{2.0 \pi}$ \\
\hline Phase deviation & 0 & $0.1 \pi$ & $0.2 \pi$ & $0.25 \pi$ & 0 & 0 & $0.1 \pi$ & $0.15 \pi$ & 0 & 0 & 0 & $0.1 \pi$ \\
\hline Boost voltage $\left(\mathrm{V}_{\mathrm{rms}}\right)$ & 0 & 4 & 12 & 15 & 0 & 0 & 4 & 8 & 0 & 0 & 0 & 3 \\
\hline$\tau_{\mathrm{r}}(\mathrm{ms})$ & 0.72 & 0.31 & 0.18 & 0.14 & 1.21 & 0.72 & 0.43 & 0.29 & 1.69 & 0.87 & 0.59 & 0.38 \\
\hline$\tau_{\mathrm{f}}(\mathrm{ms})$ & 0.21 & 0.16 & 0.15 & 0.14 & 0.32 & 0.27 & 0.25 & 0.24 & 0.42 & 0.38 & 0.40 & 0.39 \\
\hline Average $\tau_{\mathrm{r}}$ & \multirow{2}{*}{\multicolumn{4}{|c|}{$0.34 \mathrm{~ms}$}} & \multirow{2}{*}{\multicolumn{4}{|c|}{$\begin{array}{l}0.66 \mathrm{~ms} \\
0.27 \mathrm{~ms}\end{array}$}} & \multicolumn{4}{|c|}{$0.88 \mathrm{~ms}$} \\
\hline Average $\tau_{f}$ & & & & & & & & & \multicolumn{4}{|c|}{$0.40 \mathrm{~ms}$} \\
\hline
\end{tabular}

Figure 7 shows the response time of phase modulations in RM7 cells with four applied voltages and the phase deviations for $1.5 \pi$ and $2.0 \pi$ phase modulations. Compared with experimental results in the RM8 cell, the phase deviations were smaller, and the required boost voltages were also reduced. The voltages of correct phase modulations of $1.5 \pi$ and $2.0 \pi$ were achieved with applied voltages of 84 and $103 \mathrm{~V}_{\mathrm{rms}}$. Finally, the response time of phase modulations in the RM5N2 cell is shown in Figure 8, which shows only a slight phase deviation (about $0.1 \pi$ ) in the $2.0 \pi$ phase modulation, and it can be addressed with an applied voltage of $82 \mathrm{~V}_{\text {rms }}$. The electrostriction effect was insignificant because of the low phase modulation voltage in the RM5N2 cell for operating $2.0 \pi$ phase modulation. Given that polymer networks exist in PNLC cells providing more constrains, the free relaxation time $\left(\tau_{0}\right)$ is dependent on the average sizes of the LC microdomains $(D)$ and approximately satisfied the equation $\tau_{0}=\gamma_{1} D^{2} / K_{11} \pi^{2}$ [26], where $\gamma_{1}$ is LC rotational viscosity and $K_{11}$ is the LC splay elastic constant. In general, the decay time is similar to $\tau_{0}$; rising time is more complicated to illustrate but is also related to $\tau_{0}$ and applied voltage [6]. Due to larger sizes of LC microdomains generated in the RM7 cell, the average rising time (about $0.66 \mathrm{~ms}$ ) and falling time (about $0.27 \mathrm{~ms}$ ) were slower than that in the RM8 cell ( $0.34 \mathrm{~ms}$ rising time and $0.17 \mathrm{~ms}$ falling time). According to results in Figure $4 \mathrm{a}$, the average sizes of the LC microdomains in the RM5N2 cell were possibly close to that in the RM7 cell, but the average rising time $(0.88 \mathrm{~ms})$ and falling time $(0.40 \mathrm{~ms})$ were slower. It was attributed to the more NVP dopant in the RM5N2 cell that reduced the degrees of constrains between the LC molecules and the polymer networks [27].

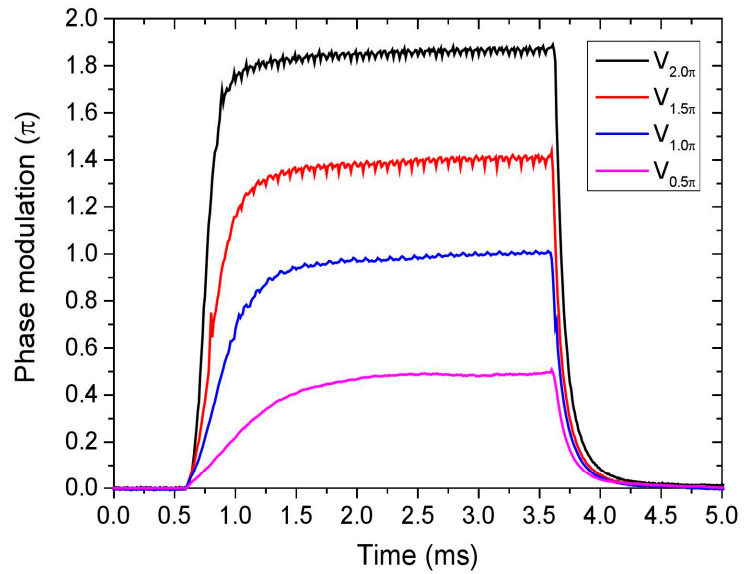

(a)

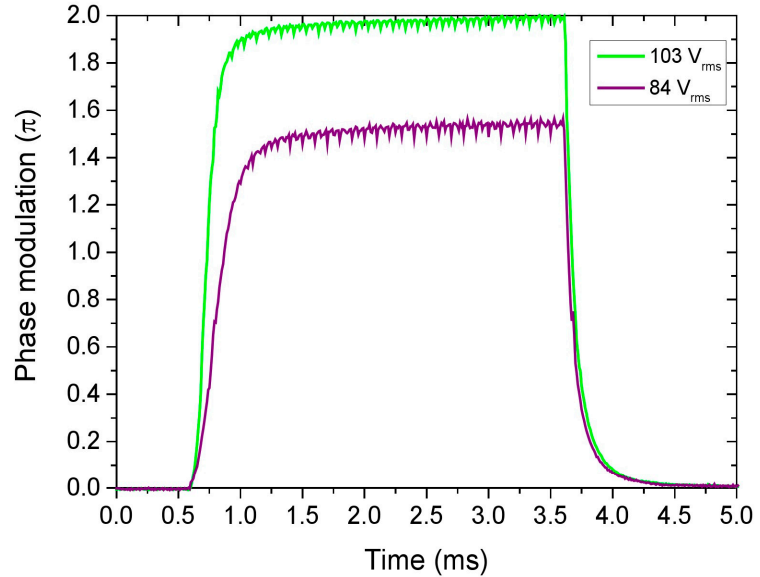

(b)

Figure 7. Measurements of response time in the RM7 cell for operating phase modulations: (a) Phase modulation voltages according to Table 2; (b) High phase modulation voltages corresponding to phase modulations of $1.5 \pi$ and $2.0 \pi$. 


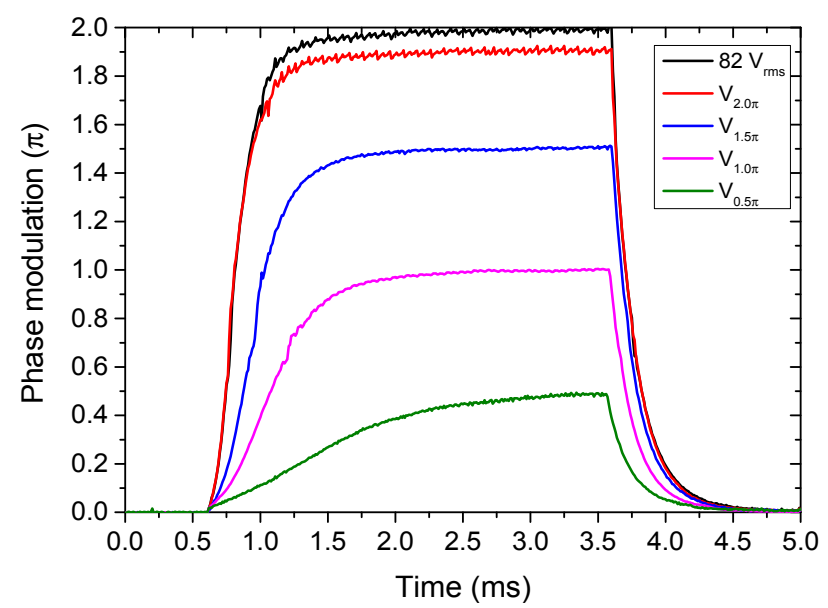

Figure 8. Measurements of response time in the RM5N2 cell for operating phase modulations.

\section{Conclusions}

We demonstrated RM257-doped PNLC cells processed via holographic exposure to realize infrared phase modulators at $\lambda=1550 \mathrm{~nm}$. As a result, the completed phase modulators possess a fast response time ( $<1 \mathrm{~ms})$ and were free of optical scattering in the range of $2 \pi$ phase modulation. However, the high operating voltages must be improved to prevent unstable phase modulation control due to the electrostriction effect. Adding more than $2 \mathrm{wt} \%$ of a mono-acrylate monomer (NVP) in PNLC cells significantly decreased the operating voltages and simultaneously maintained the phase modulators without optical scattering. Moreover, the NVP dopant also effectively improved the electrostriction effect. Finally, the optimal PNLC phase modulator was operated at a low voltage of $79 \mathrm{~V}_{\text {rms }}$ with negligible unstable phase modulation control and a fast response time (average rising and falling times of 0.88 and $0.40 \mathrm{~ms}$, respectively).

Acknowledgments: The authors would like to thank the Ministry of Science and Technology (MOST) for financially supporting this research under Grant No. MOST 104-2221-E-006-185-MY3.

Author Contributions: Chun-Yu Chien and Chia-Rong Sheu conceived the experiments. Chun-Yu Chien performed the experiments, analyzed the data, and wrote the paper. Chia-Rong Sheu supervised the study.

Conflicts of Interest: The authors declare no conflicts of interest.

\section{References}

1. Sun, J.; Wu, S.T. Recent advances in polymer network liquid crystal spatial light modulators. J. Polym. Sci. Polym. Phys. 2014, 3, 183-192. [CrossRef]

2. Hsu, C.J.; Sheu, C.R. Using photopolymerization to achieve tunable liquid crystal lenses with coaxial bifocals. Opt. Express 2012, 20, 4738-4746. [CrossRef] [PubMed]

3. Chen, H.; Peng, F.; Luo, Z.; Xu, D.; Wu, S.T.; Li, M.C.; Lee, S.L.; Tsai, W.C. High performance liquid crystal displays with a low dielectric constant material. Opt. Mater. Express 2014, 4, 2262-2273. [CrossRef]

4. Wu, S.T.; Efron, U.; Hess, L.D. Birefringence measurements of liquid crystals. Appl. Opt. 1984, 23, 3911-3915. [CrossRef] [PubMed]

5. Wu, S.T.; Wu, C.S. Experimental confirmation of the Osipov-Terentjev theory on the viscosity of nematic liquid crystals. Phys. Rev. A 1990, 42, 2219-2227. [CrossRef] [PubMed]

6. Wang, H.; Wu, T.X.; Zhu, X.; Wu, S.T. Correlations between liquid crystal director reorientation and optical response time of a homeotropic cell. J. Appl. Phys. 2004, 95, 5502-5508. [CrossRef]

7. Yu, B.H.; Song, D.H.; Kim, K.H.; Park, B.W.; Choi, S.W.; Park, S.I.; Kang, S.G.; Yoon, J.H.; Kim, B.K.; Yoon, T.H. Formation of polymer networks for fast in-plane switching of liquid crystals at low temperatures. Jpn. J. Appl. Phys. 2013, 52, 094102. [CrossRef] 
8. Lim, Y.J.; Choi, Y.E.; Lee, J.H.; Lee, G.D.; Komitov, L.; Lee, S.H. Effects of three-dimensional polymer networks in vertical alignment liquid crystal display controlled by in-plane field. Opt. Express 2014, 22, 10634-10641. [CrossRef] [PubMed]

9. Inoue, Y.; Yoshida, H.; Ozaki, M. Nematic liquid crystal nanocomposite with scattering-free, microsecond electro-optic response. Opt. Mater. Express 2014, 4, 916-923. [CrossRef]

10. Sun, J.; Xu, S.; Ren, H.; Wu, S.T. Reconfigurable fabrication of scattering-free polymer network liquid crystal prism/grating/lens. Appl. Phys. Lett. 2013, 102, 161106. [CrossRef]

11. Lorenz, A.; Braun, L.; Kolosova, V. Continuous optical phase modulation in a copolymer network nematic liquid crystal. ACS Photonics 2016, 3, 1188-1193. [CrossRef]

12. Love, G.D.; Kirby, A.K.; Ramsey, R.A. Sub-millisecond, high stroke phase modulation using polymer network liquid crystals. Opt. Express 2010, 18, 7384-7489. [CrossRef] [PubMed]

13. Chien, C.Y.; Hsu, C.J.; Chen, Y.W.; Tseng, S.H.; Sheu, C.R. Holographic polymer networks formed in liquid crystal phase modulators via a He-Ne laser to achieve ultra-fast optical response. Opt. Express 2016, 24, 7534-7542. [CrossRef] [PubMed]

14. Fan, Y.H.; Lin, Y.H.; Ren, H.; Gauza, S.; Wu, S.T. Fast-response and scattering-free polymer network liquid crystals for infrared light modulators. Appl. Phys. Lett. 2004, 84, 1233-1235. [CrossRef]

15. Peng, F.; Chen, H.; Tripathi, S.; Twieg, R.J.; Wu, S.T. Fast-response infrared phase modulator based on polymer network liquid crystal. Opt. Mater. Express 2015, 5, 265-273. [CrossRef]

16. Sun, J.; Chen, Y.; Wu, S.T. Submillisecond-response and scattering-free infrared liquid crystal phase modulators. Opt. Express 2012, 20, 20124-20129. [CrossRef] [PubMed]

17. Sun, J.; Xian, H.; Chen, Y.; Wu, S.T. Submillisecond-response polymer network liquid crystal phase modulators at 1.06-1 m wavelength. Appl. Phys. Lett. 2011, 99, 021106. [CrossRef]

18. Bunning, T.J.; Natarajan, L.V.; Tondiglia, V.P.; Sutherland, R.L. Holographic polymer-dispersed liquid crystals (H-PDLCs). Annu. Rev. Mater. Sci. 2000, 30, 83-115. [CrossRef]

19. Sio, L.D.; Tabiryan, N.; Bunning, T.J. POLICRYPS-based electrically switchable Bragg reflector. Opt. Express 2015, 23, 32696-32702. [CrossRef] [PubMed]

20. Zhao, X.J.; Liu, C.L.; Duan, J.Z.; Zeng, J.C.; Zhang, D.Y.; Luo, Y.Q. Morphology effect on the light scattering and dynamic response of polymer network liquid crystal phase modulator. Opt. Express 2014, 22, 14757-14768.

21. Lan, Y.F.; Tsai, C.Y.; Lu, J.K.; Sugiura, N. Mechanism of hysteresis in polymer-network stabilized blue phase liquid crystal. Polymer 2013, 54, 1876-1879. [CrossRef]

22. Chien, C.Y.; Sheu, C.R. Small dosage of holographic exposure via a He-Ne laser to fabricate tunable liquid crystal phase gratings operated with low electric voltages. Liq. Cryst. 2017, 44, 854-862. [CrossRef]

23. Hsu, C.C.; Chen, Y.H.; Li, H.W.; Hsu, J.S. Low switching voltage ZnO quantum dots doped polymer-dispersed liquid crystal film. Opt. Express 2016, 24, 7063-7068. [CrossRef] [PubMed]

24. Ma, R.Q.; Yang, D.K. Freedericksz transition in polymer-stabilized nematic liquid crystals. Phys. Rev. E Stat. Phys. Plasmas Fluids Relat. Interdiscip. Topics 2000, 61, 1567-1573. [CrossRef] [PubMed]

25. Yang, D.K.; Cui, Y.; Nemati, H.; Zhou, X.; Moheghi, A. Modeling aligning effect of polymer network in polymer stabilized nematic liquid crystals. J. Appl. Phys. 2013, 114, 243515. [CrossRef]

26. Sun, J.; Wu, S.T.; Haseba, Y. A low voltage submillisecond-response polymer network liquid crystal spatial light modulator. Appl. Phys. Lett. 2014, 104, 023305. [CrossRef]

27. Nie, X.; Lu, R.; Xian, H.; Wu, T.X.; Wu, S.T. Anchoring energy and cell gap effects on liquid crystal response time. J. Appl. Phys. 2007, 101, 103110. [CrossRef]

(C) 2017 by the authors. Licensee MDPI, Basel, Switzerland. This article is an open access article distributed under the terms and conditions of the Creative Commons Attribution (CC BY) license (http://creativecommons.org/licenses/by/4.0/). 